\title{
Discrete Wavelets Associated with DUNKL Operator on Real Line
}

\author{
C. P. Pandey \\ Department of Mathematics \\ Ajay Kumar Garg Engineering, \\ Ghaziabad (India)
}

\author{
Rakesh Mohan \\ Department of Mathematics \\ DIT University, Dehradun \\ (India)
}

\author{
Bhairaw Nath Tripathi \\ Department of Mathematics \\ Noida Institute of Engineering \\ \& Technology, Greater Noida \\ (India)
}

\begin{abstract}
Using convolution theory of the dunkl transform, discrete dunkl wavelet transform is defined. A reconstruction formula for the discrete dunkl wavelet is obtained. Important properties of the discrete dunkl wavelet are presented. Frames and Riesz basis involving dunkl wavelets are studied.
\end{abstract}

\section{Keywords}

Dunkl transform, wavelet transform, Dunkl operator.

AMS Subject Classifications: 42C40, 65T60, 44A35,65R10

\section{INTRODUCTION}

The wavelet transform of a function $f \in L^{2}(R)$ with respect to the wavelet

$$
\begin{aligned}
& \psi \in L^{2}(R)_{\text {is }} \psi \in L^{2}(R)_{\text {defined by }} \\
& \left(W_{\psi} f\right)(b, a)=\int_{-\infty}^{\infty} f(t) \overline{\psi_{b, a}(t)} d t, a, b \in R, a>0,
\end{aligned}
$$

$$
\text { where } \psi_{b, a}(t)=a^{-1 / 2} \psi\left(\frac{t-b}{a}\right) .
$$

In terms of translation $\tau \mathrm{b}$ defined by

$$
\tau_{b} \psi(t)=\psi(t-b), \quad \mathrm{b}_{\in_{\mathrm{R}}}
$$

and dilation Da defined by

$$
D_{a} \psi(t)=a^{-1 / 2} \psi\left(\frac{t}{a}\right), \quad \mathrm{a}>0
$$

we can write $\psi_{b, a}(t)=\tau_{b} D_{a} \psi(t)$

From (1) and (3) it is clear that wavelet transform of the function $\mathrm{f}$ on $\mathrm{R}$ is an integral transform for which the kernel is the dilated translate of $\psi$.

We can also express (1) as the convolution:

$$
\begin{aligned}
& \left(W_{\psi} f\right)(\mathrm{b}, \mathrm{a})=\left(f * \mathrm{~g}_{\mathrm{o}, \mathrm{a}}\right)(\mathrm{b}) \\
& \text { where } \\
& g(t)=\overline{\psi(-t) .}
\end{aligned}
$$

$$
\Lambda_{\alpha}(f)(x)=\lambda f(x), f(0)=1, x \in R,
$$

where

$$
\begin{gathered}
\Lambda_{\alpha}(f)(x)= \\
\frac{d}{d x} f(x)+\frac{2 \alpha+1}{x}\left(\frac{f(x)-f(-x)}{2}\right) .
\end{gathered}
$$

called Dunkl Operator has a unique solution $E_{\alpha}(\lambda x)$ called Dunkl kernel and given by

$$
\begin{aligned}
& E_{\alpha}(\lambda x)=j_{\alpha}(i \lambda x)+\frac{\lambda x}{2(\alpha+1)} j_{\alpha+1}(i \lambda x), \\
& x \in R
\end{aligned}
$$

where $j_{\alpha}$ is the normalized Bessel function of the first kind and order $\alpha$ defined by

$$
j_{\alpha}(z)=2^{\alpha} \Gamma(\alpha+1) \frac{J_{\alpha}(z)}{z^{\alpha}}=\Gamma(\alpha+1) \sum_{n=0}^{\infty} \frac{(-1)^{n}(z / 2)^{2 n}}{n ! \Gamma(n+\alpha+1)}
$$$$
z \in C \text {. }
$$

We can write for $x \in R$ and $\lambda \in C$

$$
E_{\alpha}(-i \lambda x)=\frac{\Gamma(\alpha+1)}{\sqrt{\pi} \Gamma(\alpha+1 / 2)} \int_{-1}^{1}\left(1-t^{2}\right)^{\alpha-1 / 2}(1-t) e^{i \lambda \lambda t} d t
$$

Let $\alpha>-1 / 2$ be a fixed number and $\mu_{\alpha}$ be the weighted Lebesgue measure on $\mathrm{R}$, given by

$d \mu_{\alpha}(x):=\left(2^{\alpha+1} \Gamma(\alpha+1)\right)^{-1}|x|^{2 \alpha+1} d x$

For every $1 \leq p \leq \infty$, we denote by $L_{p, \alpha}=L_{p}\left(d \mu_{\alpha}\right)$ the space of complex-valued functions $\mathrm{f}$, measurable on $\mathrm{R}$ such that

For $\alpha \geq-1 / 2$ and $\lambda \in C$, the initial value problem 
$\|f\|_{p, \alpha}=\left(\int_{R}|f(x)|^{p} d \mu_{\alpha}(x)\right)^{1 / p}<\infty$ if $p \in[1, \infty)$

The Dunkl kernel gives rise to an integral transform, called Dunkl transform on $\mathrm{R}$, which was introduced and studied in [5].

The Dunkl transform $F_{\alpha}$ of a function $f \in L_{1, \alpha}(R)$, is given by

$$
\begin{aligned}
& F_{\alpha} f(\lambda)=\hat{f}(\lambda) \\
& =\int_{R} E_{\alpha}(-i \lambda x) f(x) d \mu_{\alpha}(x) ; \lambda \in R
\end{aligned}
$$

An inversion formula for this transform is given by

$$
\begin{aligned}
& F_{\alpha}^{-1}(\hat{f}(\lambda))=(\hat{f}(\lambda))^{\vee} \\
& =f(x)=\int_{R} E_{\alpha}(i \lambda x) \hat{f}(\lambda) d \mu_{\alpha}(\lambda)
\end{aligned}
$$

An Parseval formula for this transform is given by

$$
\int_{-\infty}^{\infty} f(x) g(x) d x=\int_{-\infty}^{\infty} \hat{f}(\lambda) \hat{g}(\lambda) d \mu_{\alpha}(\lambda)
$$

\section{DUNKL TRANSLATION AND}

\section{CONVOLUTION}

In this section following [5] we define Dunkl translation and associated convolution and discuss their important properties.

To define Dunkl convolution ${ }^{*} \alpha$ we need to introduce a special type of translation, called Dunkl translation. For this purpose we need the basic function

$$
W_{\alpha}(x, y, z)=\left(1-\sigma_{x, y, z}+\sigma_{z, x, y}+\sigma_{z, y, x}\right) \Delta_{\alpha}(x, y, z)
$$

Where

$$
\sigma_{x, y, z}=\left\{\begin{array}{cc}
\frac{x^{2}+y^{2}+z^{2}}{2 x y}, \text { if } & x, y \in R \backslash 0 \\
0 & \text { otherwise }
\end{array}\right.
$$

And $\Delta_{\alpha}$ is the Bessel kernel given by

$\Delta_{\alpha}(x, y, z)= \begin{cases}d_{\alpha} \frac{\left(\left[(|x|+|y|)^{2}-z^{2}\right]\left[z^{2}-(|x|-|y|)^{2}\right]\right)^{\alpha-1 / 2}}{|x y z|^{2 \alpha}}, & \text { if }|z| \in A_{x y}, \\ 0 & \text { otherwise, }\end{cases}$

where

$$
d_{\alpha}=(\Gamma(\alpha+1))^{2} /\left(2^{\alpha-1} \sqrt{\pi} \Gamma\left(\alpha+\frac{1}{2}\right)\right)
$$

and

$$
A_{x, y}=(|| x|-| y||,|x|+|y|)
$$

Also

$$
\int_{R}\left|W_{\alpha}(x, y, z)\right| d \mu_{\alpha}(z) \leq 4 \text {. }
$$

The Dunkl translation $\tau_{x} f(y)$ of $f \in L_{p, \alpha}(R)$, $1 \leq p<\infty$ is defined as follows

$$
\tau_{x} f(y)=f(x, y)=\int_{R} f(z) W_{\alpha}(x, y, z) d \mu_{\alpha}(z)
$$

Lemma 1. For all $x \in R$ and $f \in L_{p, \alpha}(R), p \geq 1$

$\left\|\tau_{x} f\right\|_{p, \alpha} \leq 4\|f\|_{p, \alpha}$

$\left(\tau_{x} f\right)^{\wedge}(\lambda)=E_{\alpha}(i \lambda x) f^{\wedge}(\lambda)$

Let $p, q, r \in\left[1, \infty\left[\right.\right.$ and $\frac{1}{r}=\frac{1}{p}+\frac{1}{q}-1$. Then Dunkl convolution of $f \in L_{p, \alpha}(R)$ and $g \in L_{q, \alpha}(R)$ is defined by

$$
f *_{\alpha} g(x)=\int_{R} \tau_{x} f(-y) g(y) d \mu_{\alpha}(y)
$$

Lemma 2. Let $p, q, r \in\left[1, \infty\left[\right.\right.$ and $\frac{1}{r}=\frac{1}{p}+\frac{1}{q}-1$ $f \in L_{p, \alpha}(R)$ and $g \in L_{q, \alpha}(R)$. Then convolution $f^{*}{ }_{\alpha} g(x)_{\text {satisfies }}$ the following norm inequality

$$
\left\|f{ }_{\alpha}^{*} g\right\|_{r, \alpha} \leq 4\|f\|_{p, \alpha}\|g\|_{q, \alpha},
$$

Moreover for all $f \in L_{1, \alpha}(R)$ and $g \in L_{2, \alpha}(R)$, we have

(ii)

$$
\left(f{ }_{\alpha}^{*} g\right)^{\wedge}(\lambda)=f^{\wedge}(\lambda) g^{\wedge}(\lambda)
$$

\section{DUNKL WAVELET TRANSFORM}

For a function $\psi \in L_{p, \alpha}(R)$, define the dilation Da is given by

$$
D_{a} \psi(x)=\psi(a x), \quad \mathrm{a} \in \mathrm{R}
$$

Using the Dunkl translation and the above dilation, the Dunkl wavelet $\psi_{b, a}(x)$ is defined as follows 


$$
\begin{aligned}
& \psi_{b, a}(x)=\tau_{b} D_{a} \psi(x)=\tau_{\mathrm{b}} \psi(a x) \\
& =\tau_{\mathrm{b}} \psi(a x) \\
& \int_{R} \psi(a z) W_{\alpha}(b, x, z) d \mu_{\alpha}(z), \quad \mathrm{b} \in \mathrm{R} .
\end{aligned}
$$

The integral is convergent by virtue of (18). Now, using the wavelet $\psi_{b, a}$ the Dunkl wavelet transform (DWT) of $f \in L_{q, \alpha}, \frac{1}{\mathrm{p}}+\frac{1}{q}=1$,

$$
\begin{aligned}
& \left(D_{\psi} f\right)(\mathrm{b}, \mathrm{a})=\left\langle f(\mathrm{x}), \psi_{\mathrm{b}, \mathrm{a}}(x)\right\rangle \\
& =\int_{-\infty}^{\infty} f(x) \overline{\psi_{b, a}(x)} d \mu_{\alpha} \\
& =\int_{-\infty}^{\infty} \int_{-\infty}^{\infty} f(x) \overline{\psi(a z)} W_{\alpha}(b, x, z) d \mu_{\alpha}(z) d \mu_{\alpha}(x)
\end{aligned}
$$

Provided the integral is convergent. Since by (17) and (18) $\psi_{b, a}(x) \in L_{p, \alpha}(R)_{\text {whenever }} \psi \in L_{p, \alpha}(R)$ virtue of Lemma, the integral is convergent for $f \in L_{q, \alpha}, \frac{1}{p}+\frac{1}{q}=1$

\section{THE DISCRETE DUNKL WAVELET TRANSFORM}

In the continuous Dunkl wavelet transform (25), if we discretize only the dilation parameter a by assuming that aj = $2-\mathrm{j}, \mathrm{j} \in \mathrm{Z}$, and the translation parameter $\mathrm{b}$ is allowed to vary over all of $\mathrm{R}$, then the transform so obtained is called semidiscrete Dunkl wavelet transform. If we discretize the translation parameter $\mathrm{b}$ also by restricting it to the discrete set of points:

$$
b_{j, k}=\frac{k}{2^{j}} b_{0}, j \in \mathbf{Z}, k \in \mathbf{N}_{0},
$$

where b0 $>0$ is a fixed constant, we get the discrete Dunkl wavelet transform. We shall use the notation:

$$
\psi_{\mathrm{b}_{0} ; \mathrm{j}, \mathrm{k}}(\mathrm{t})=\psi_{\mathrm{b} j \mathrm{j}, \mathrm{i} ; \mathrm{j}_{\mathrm{j}}}(\mathrm{t})=\psi\left(2^{-\mathrm{j}} \mathrm{t}, 2^{-\mathrm{j}} \mathrm{k} \mathrm{b}_{0}\right)
$$

Then the discrete Dunkl wavelet transform of any $f \in L_{2, \alpha}(\mathrm{R})$

$$
\left(\mathrm{D}_{\psi} f\right)\left(\mathrm{b}_{\mathrm{j}, \mathrm{k}, \mathrm{a}_{\mathrm{j}}}\right)=<f, \psi_{b_{0} ; j, k}>, j \in \mathbf{Z}, k \in \mathbf{N}_{0}
$$

The stability condition for this reconstruction takes the form
$A\|f\|_{2}^{2} \leq \sum_{K \in N_{0}}\left|<\mathrm{f}, \psi_{\mathrm{b}_{0} ; j, k}>\right|^{2} \leq \mathrm{B}\|\mathrm{f}\|_{2}^{2}, \mathrm{f} \in L_{2, \alpha}(\mathrm{R})$

In what follows we assume that $\psi \in L_{1, \alpha} \cap L_{2, \alpha}$ satisfies, the so called, "stability condition"

$$
\mathrm{A} \leq \sum_{\mathrm{j}=-\infty}^{\infty} \mid \hat{\psi}\left(\left.2^{-\mathrm{j}} \lambda\right|^{2} \leq \mathrm{B}\right. \text { a.e. }
$$

for certain positive constants $\mathrm{A}$ and $\mathrm{B}, 0<A \leq B<\infty$. The function $\psi \in L_{1, \alpha} \cap L_{2, \alpha}$ satisfying (29) is called dyadic wavelet. Using the definition (25) we define the semidiscrete Dunkl wavelet transform of any $f \in L_{1, \alpha} \cap L_{2, \alpha}$ by

$$
\begin{gathered}
\left(\mathrm{D}_{j}^{\psi} f\right)(\mathrm{b})=\left(\mathrm{D}_{\psi} f\right)\left(\mathrm{b}, 2^{-\mathrm{j}}\right) \\
=\int_{-\infty}^{\infty} f(t) \overline{\psi_{b, 2^{-j}}(t)} \mathrm{d} \mu_{\alpha}(\mathrm{t}) \\
=\int_{-\infty}^{\infty} f(t) \overline{\psi\left(2^{-j} t, b\right)} \mathrm{d} \mu_{\alpha}(\mathrm{t})(30) \\
=\left(\mathrm{f} * \bar{\psi}_{\mathrm{j}}\right)_{\mathrm{j} \in \mathrm{Z}}, \\
\text { where } \psi_{j}(z)=\psi\left(2^{-\mathrm{j}} z\right), \mathrm{j} \in \mathrm{Z} .
\end{gathered}
$$

Theorem1. Assume that the semi-discrete Dunkl wavelet transform of any $f \in L_{1, \alpha} \cap L_{2, \alpha}$ is defined by (32). Let us define another wavelet $\square *$ by means of its Dunkl transform,

$$
\hat{\psi^{*}}(\lambda)=\frac{\hat{\psi}(\lambda)}{\sum_{\mathrm{k}=-\infty}^{\infty}\left|\hat{\psi}\left(2^{-\mathrm{k}} \lambda\right)\right|^{2}}
$$

Then

$$
f(t)=\sum_{\mathrm{j}=-\infty}^{\infty} \int_{-\infty}^{\infty}\left(\mathrm{D}_{\mathrm{j}}^{\psi} f\right)(\mathrm{b})\left(\hat{\psi}^{*}\left(2^{-\mathrm{j}} \lambda\right) \mathrm{E}_{\alpha}(i \lambda t)\right)^{\vee}(b) d \mu_{\alpha}(b) .
$$

Proof. For any $f \in L_{1, \alpha} \cap L_{2, \alpha}$ we have

$$
\begin{aligned}
& \sum_{j=-\infty}^{\infty} \int_{-\infty}^{\infty}\left(D_{j}^{\psi} f\right)(b)\left(\hat{\psi}^{*}\left(2^{-j} \lambda\right) \mathrm{E}_{\alpha}(\mathrm{i} \lambda t)\right)^{v}(b) \mathrm{d} \mu(\mathrm{b}) \\
& =\sum_{j=-\infty}^{\infty} \int_{-\infty}^{\infty}\left(D_{j}^{\psi} f\right)^{\wedge}(\lambda) \hat{\psi}^{*}\left(2^{-j} \lambda\right) \mathrm{E}_{\alpha}(\mathrm{i} \lambda t) \mathrm{d} \mu_{\alpha}(\lambda)
\end{aligned}
$$




$$
\begin{aligned}
& =\sum_{j=-\infty}^{\infty} \int_{-\infty}^{\infty} \hat{f}(\lambda) \overline{\hat{\psi}\left(2^{-j} \lambda\right)} \hat{\psi^{*}\left(2^{-\mathrm{j}} \lambda\right) \mathrm{E}_{\alpha}(\mathrm{i} \lambda t) d \mu_{\alpha}(\lambda)} \\
& =\sum_{j=-\infty}^{\infty} \int_{-\infty}^{\infty} \hat{f}(\lambda) \overline{\hat{\psi}\left(2^{-j} \lambda\right)} \frac{\hat{\psi\left(2^{-j} \lambda\right)}}{\sum_{k=-\infty}^{\infty}\left|\hat{\psi}\left(2^{-k} 2^{-j} \lambda\right)\right|^{2}} \mathrm{E}_{\alpha}(\mathrm{i} \lambda t) d \mu_{\alpha}(\lambda) \\
& =\int_{-\infty}^{\infty} \hat{\mathrm{f}}(\lambda) \mathrm{E}_{\lambda}(\mathrm{i} \lambda t) \mathrm{d} \mu_{\alpha}(\lambda) \\
& =\mathrm{f}(\mathrm{t}) .
\end{aligned}
$$

The above theorem leads to the following definition of dyadic dual.

Definition1. A function $\tilde{\psi} \in L_{2, \alpha}(R)$ is called a dyadic dual of a dyadic wavelet $\square \square$, if every f $\in L_{2, \alpha}(\mathrm{R})$ can be expressed as

$$
f(t)=\sum_{\mathrm{j}=-\infty}^{\infty} \int_{-\infty}^{\infty}\left(D_{\mathrm{j}}^{\psi} f\right)(b)\left(\tilde{\psi}\left(2^{-j} \lambda\right) \mathrm{E}_{\alpha}(\mathrm{i} \lambda t)\right)^{\vee}(b) d \mu(b)
$$

Theorem2. Assume that the discrete Dunkl wavelet transform of any $\mathrm{f} \in L_{2, \alpha}(\mathrm{R})$ is defined by (27) and stability condition (28) holds. Let $\mathrm{T}$ be a linear operator on $L_{2, \alpha}(\mathrm{R})$ defined by

$$
\mathrm{Tf}=\sum_{\substack{\mathrm{j} \in \mathrm{Z} \\ \mathrm{K} \in \mathrm{N}_{0}}}<\mathrm{f}, \psi_{\mathrm{b}_{0} ; \mathrm{j}, \mathrm{k}}>\psi_{\mathrm{b}_{0} ; \mathrm{j}, \mathrm{k}}
$$

Then

$$
\begin{aligned}
f & =\Sigma<f, \psi_{b_{0} ; j, k}>\psi_{b_{0}}^{j, k}, \\
\text { where } \psi_{b_{0}}^{j, k} & =T^{-1} \psi_{b_{0} ; j, k} ; j \in Z .
\end{aligned}
$$

Proof. From the condition (28), it follows that the operator defined by (35) is a one-one bounded linear operator. Set

$$
\mathrm{g}=\mathrm{T} \mathrm{f}, \quad f \in L_{2, \alpha}(\mathrm{R})
$$

Then, we have

$$
<\mathrm{Tf}, \mathrm{f}>=\sum_{\substack{\mathrm{j} \in \mathrm{Z} \\ \mathrm{K} \in \mathrm{N}_{0}}}\left|<\mathrm{f}, \psi_{\mathrm{b}_{0} ; \mathrm{j}, \mathrm{k}}>\right|^{2}
$$

Therefore,

$A\left\|T^{-1} g\right\|_{2}^{2}=A\|f\|_{2}^{2} \leq$

$<\mathrm{Tf}, \mathrm{f}>=<\mathrm{g}, \mathrm{T}^{-1} g>\leq\|g\|_{2}\left\|T^{-1} g\right\|_{2}$

so that
$\left\|\mathrm{T}^{-1} \mathrm{~g}\right\| \leq \frac{1}{\mathrm{~A}}\|\mathrm{~g}\|_{2}$

Hence every $f \in L_{2, \alpha}(R)$ an be reconstructed from its discrete Dunkl wavelet transform given by (27). Thus

$$
\mathrm{f}=\mathrm{T}^{-1} \mathrm{Tf}=\sum_{\substack{\mathrm{j} \in \mathrm{Z} \\ \mathrm{k} \in \mathrm{N}_{0}}}<\mathrm{f}, \psi_{\mathrm{b}_{0} ; \mathrm{j}, \mathrm{k}}>\mathrm{T}^{-1} \psi_{\mathrm{b}_{0} ; \mathrm{j}, \mathrm{k}}
$$

Finally, set

$$
\psi_{b_{0}}^{j, k}=T^{-1} \psi_{b_{0} ; j, k} ; j \in \mathbf{Z}, k \in \mathbf{N}_{0}
$$

Then, the reconstruction (37) can be expressed as follows:

$$
\mathrm{f}=\sum_{\substack{\mathrm{j} \in \mathrm{Z} \\ \mathrm{k} \in \mathrm{N}_{0}}}<\mathrm{f}, \psi_{\mathrm{b}_{0} ; \mathrm{j}, \mathrm{k}}>\psi_{\mathrm{b}_{0}}^{\mathrm{j}, \mathrm{k}}
$$

\section{FRAMES AND RIESZ BASIS IN \\ $L_{2, \alpha}(\mathrm{R})$}

In this section, using $\psi_{\mathrm{b}_{0} ; \mathrm{j}, \mathrm{k}}$ a frame is defined and Riesz basis of $f \in L_{2, \alpha}(\mathrm{R})$ is studied.

Definition 2. A function $\psi \in \mathrm{L}^{2}(\mu)$ is said to generate a frame $\left\{\psi_{b o ; j, k}\right\} \quad$ of $\mathrm{f} \in L_{2, \alpha}(\mathrm{R})$ with sampling rate b0 if (28) holds for some positive constants A and B. If A = B, then the frame is called a tight frame.

Definition3. A function $\psi \in L_{2, \alpha}(\mathrm{R})$ is said to generate a Riesz basis $\left\{\psi_{\mathrm{b}_{0} ; \mathrm{j}, \mathrm{k}}\right\}$ with sampling rate b0 if the following two properties are satisfied.

(i) The linear span $\left\langle\psi_{b_{0} ; j, k}: j \in N_{0}\right\rangle$ is dense in $\psi \in L_{2, \alpha}(\mathrm{R})$

(ii) There exist positive constants $\mathrm{A}$ and $\mathrm{B}$, with $0<\mathrm{A} \leq \mathrm{B}<\infty$ such that

$$
A\left\|\left\{c_{j, k}\right\}\right\|_{\ell^{2}}^{2} \leq\left\|\sum_{\substack{j \in N_{0} \\ k \in N_{0}}} c_{j, k} \psi_{\mathrm{b}_{0} ; \mathrm{j}, \mathrm{k}}\right\|_{2}^{2} \leq \mathrm{B}\left\|\left\{\mathrm{c}_{\mathrm{j}, \mathrm{k}}\right\}\right\|_{\ell^{2}}^{2}
$$

for all $\left\{c_{j, k}\right\} \in \ell^{2}\left(N_{0}^{2}\right)$. Here A and B are called the Riesz bounds of $\left\{\psi_{\mathrm{b} 0 ; \mathrm{j}, \mathrm{k}}\right\}$.

Theorem3. Let $\psi \in L_{2, \alpha}(\mathrm{R})$ and b0 $>0$, then the following two statements are equivalent. 
(i) $\quad\left\{\psi_{\mathrm{bo} ; \mathrm{j}, \mathrm{k}}\right\}$ is a Riesz basis of $\in L_{2, \alpha}(\mathrm{R})$;

$$
\left\{\psi_{\mathrm{b}_{0} ; \mathrm{j}, \mathrm{k}}\right\} \text { is a frame of } L_{2, \alpha}(\mathrm{R}) \text { and is also an } l^{2}
$$

linearly independent family in the sense that if

$$
\begin{aligned}
& \sum \psi_{\mathrm{b}_{0} ; \mathrm{j}, \mathrm{k}} c_{j, k}=0 \text { and } \\
& \left\{\mathrm{c}_{\mathrm{j}, \mathrm{k}}\right\} \in \ell^{2}, \text { then } \mathrm{c}_{\mathrm{j}, \mathrm{k}}=0
\end{aligned}
$$

Furthermore, the Riesz bounds and frame bounds agree.

Proof. It follows from (39) that any Riesz basis is $\mathrm{l}^{2}$ - linearly independent. Let $\left\{\psi_{b o ; j, k}\right\}_{\text {be a Riesz basis with Reisz }}$ bounds A and B, and consider the "Matrix operator"

$$
\mathbf{M}=\left\lfloor\gamma_{\ell, \mathrm{m}, \mathrm{j}, \mathrm{k}}\right\rfloor_{(\ell, \mathrm{m}),(\mathrm{j}, \mathrm{k}) \in \mathrm{N}_{0} \times \mathrm{N}_{0},}
$$

where the entries are defined by

$$
\gamma_{\ell, \mathrm{m}, \mathrm{j}, \mathrm{k}}=\left\langle\psi_{\mathrm{b}_{0} ; \ell, \mathrm{m}}, \psi_{\mathrm{b}_{0} ; \mathrm{j}, \mathrm{k}}\right\rangle
$$

Then from (39), we have

$$
\begin{aligned}
& A\left\|\left\{c_{j, k}\right\}\right\|_{\ell^{2}}^{2} \leq \\
& \sum_{\ell, \mathrm{m}, \mathrm{j}, \mathrm{k}} \mathrm{c}_{\ell, m} \gamma_{\ell, m ; \ell, k} c_{j, k} \leq \mathrm{B}\left\|\left\{\mathrm{c}_{\mathrm{j}, \mathrm{k}}\right\}\right\|_{\ell^{2}}^{2}
\end{aligned}
$$

so that $\mathrm{M}$ is positive definite. We denote the inverse of $\mathrm{M}$ by

$$
\mathbf{M}^{-1}=\left[\mu_{\ell, \mathrm{m}, \mathrm{j}, \mathrm{k}}\right]_{(\ell, \mathrm{m}),(\mathrm{j}, \mathrm{k}) \in \mathrm{N}_{0}^{2}}
$$

which means that both

$$
\sum_{\gamma, s} \mu_{\ell, m, r, s} \gamma_{r, s, j, k}=\delta_{\ell, j} \delta_{\mathrm{m}, \mathrm{k}} \quad \ell, \mathrm{m}, \mathrm{j}, \mathrm{k} \in N_{0}
$$

$$
\begin{aligned}
& B^{-1}\left\|\left\{c_{j, k}\right\}\right\|_{\ell^{2}}^{2} \leq \\
& \sum_{\text {and }} \mathrm{c}_{\ell, \mathrm{m}, \mathrm{j}, \mathrm{m}} \mu_{\ell, m} \mu_{\ell, m, j, k} \bar{c}_{j, k} \leq A^{-1}\left\|\left\{c_{j, k}\right\}\right\|_{\ell^{2}}^{2}
\end{aligned}
$$

are satisfied. This allows us to introduce

$\psi^{\ell, \mathrm{m}}(\mathrm{x})=\sum_{\mathrm{j}, \mathrm{k}} \mu_{\ell, \mathrm{m}, \mathrm{j}, \mathrm{k}} \psi_{\mathrm{bo} ; \mathrm{j}, \mathrm{k}}(\mathrm{x})$

Clearly, $\quad \psi^{\ell, m} \in L_{2, \alpha}(\mathrm{R})$ and it follows from (40) and (42) that

$\left\langle\psi^{\ell, m} ; \psi_{b_{0} ; j, k}\right\rangle=\delta_{\ell, j} \delta_{\mathrm{m}, \mathrm{k}} \quad \ell, \mathrm{m}, \mathrm{j}, \mathrm{k} \in N_{0}$ which means that $\left\{\psi^{\ell, \mathrm{m}}\right\}$ is the basis of $L_{2, \alpha}(\mathrm{R})$ which is dual to $\left\{\psi_{\mathrm{b}_{0} ; \mathrm{j}, \mathrm{k}}\right\}$.

Furthermore, from (42) and (44); we conclude that

$$
\left\langle\psi^{\ell, \mathrm{m}}, \psi^{\mathrm{j}, \mathrm{k}}\right\rangle=\mu_{\ell, \mathrm{m}, \mathrm{j}, \mathrm{k}}
$$

and the Riesz bounds of $\left\{\psi^{\ell, \mathrm{m}}\right\} \operatorname{areB}^{-1}$ and $\mathrm{A}^{-1}$

In particular, for any $f \in L_{2, \alpha}(\mathrm{R})$ we may write

$$
\mathrm{f}(\mathrm{x})=\sum_{\mathrm{j}, \mathrm{k}}<\mathrm{f}, \psi_{\mathrm{b}_{0} ; \mathrm{j}, \mathrm{k}}>\psi^{\mathrm{j}, \mathrm{k}}(\mathrm{x})
$$

and

$$
\mathrm{B}^{-1} \sum_{\mathrm{j}, \mathrm{k}}\left|<\mathrm{f}, \psi_{\mathrm{b}_{0} ; \mathrm{j}, \mathrm{k}}>\right|^{2} \leq\|\mathrm{f}\|_{2}^{2} \leq \mathrm{A}^{-1} \sum_{\mathrm{j}, \mathrm{k}} \mathrm{k} \mathrm{f}, \psi_{\mathrm{b}_{0} ; \mathrm{j}, \mathrm{k}}>\left.\right|^{2} .
$$

Since, (45) is equivalent to (28) therefore, statement (i) implies statement (ii). To prove the converse part, we recall Theorem 2 and we have for any $g \in L_{2, \alpha}(\mathrm{R})$ and $\mathrm{f}=T^{-1} g$,

$$
g(x)=\sum<f, \psi_{b_{0}, j ; k}>\psi_{b_{0} j ; k}
$$

Also, by the $\mathrm{l}^{2}$ linear independence of $\left\{\psi_{\mathrm{b}_{0} \mathrm{j} ; \mathrm{k}}\right\}$, this representation is unique. From the Banach-Steinhaus and open mapping theorem it follows that $\left\{\psi_{\mathrm{b}_{0}, \mathrm{j} ; \mathrm{k}}\right\}$ is Riesz basis of $L_{2, \alpha}(\mathrm{R})$

\section{REFERENCES}

[1] C.K. Chui, An Introduction to Wavelets, Academic Press, New York (1992)

[2] Lokenath Debnath, Wavelet Transforms and their Applications, PINSA -A 6(1998), 685-713

[3] U.Depczynski, Sturm-Liouville wavelets, Applied and Computational Harmonic Analysis, 5(1998), 216-247.

[4] R.S. Pathak and M.M. Dixit, Continuous and discrete Bessel wavelet transforms, J. Computational and Applied Mathematics, 160 (2003), 241-250.

[5] Vagif S.GULIYEV and Yagub Y.MAMMADOV, Function Spaces and Integral Operators for The Dunkl Operators on the Real Line, Khajar Journal of Mathematics 4 (2006), 17-42.

[6] C.P.Pandey, Rakesh Mohan and B.N.Tripathi, Continuous Dunkl wavelet transform, International Journal of Current Engineering and technology, Vol 4, No.1, 2014 